\title{
The DNA Methylomes of Serous Borderline Tumors Reveal Subgroups With Malignant- or Benign-Like Profiles
}

\author{
Constanze Zeller, ${ }^{*}$ Wei Dai, ${ }^{*}$ Edward Curry, ${ }^{*}$ Afshan Siddiq, ${ }^{\dagger}$ Andrew Walley, ${ }^{\dagger}$ Nahal Masrour, ${ }^{*}$ Isidora Kitsou-Mylona, ${ }^{\ddagger}$ \\ Gail Anderson, ${ }^{*}$ Sadaf Ghaem-Maghami, ${ }^{\S}$ Robert Brown, ${ }^{*}$ and Mona El-Bahrawy ${ }^{\ddagger \uparrow}$
}

From the Epigenetics Unit,* Department of Surgery and Cancer, the Department of Genomics of Common Disease, ${ }^{\dagger}$ School of Public Health, and the Departments of Histopathology $y^{\ddagger}$ and Surgery and Cancer, ${ }^{\S}$ Imperial College London, Hammersmith Campus, London, United Kingdom; and the Department of Pathology, "Faculty of Medicine, University of Alexandria, Egypt

Accepted for publication

November 15, 2012.

Address correspondence to Mona El-Bahrawy, Ph.D., Department of Histopathology, Imperial College London, Hammersmith Hospital, Du Cane Road, London W12 0HS, United Kingdom. E-mail: m.elbahrawy@imperial.ac.uk.

\begin{abstract}
Serous borderline tumors (SBOTs) are a challenging group of ovarian tumors positioned between benign and malignant disease. We have profiled the DNA methylomes of 12 low-grade serous carcinomas (LGSCs), 19 SBOTs, and 16 benign serous tumors (BSTs) across 27,578 CpG sites to further characterize the epigenomic relationship between these subtypes of ovarian tumors. Unsupervised hierarchical clustering of DNA methylation levels showed that LGSCs differ distinctly from BSTs, but not from SBOTs. Gene ontology analysis of genes showing differential methylation at linked CpG sites between LGSCs and BSTs revealed significant enrichment of gene groups associated with cell adhesion, cell-cell signaling, and the extracellular region, consistent with a more invasive phenotype of LGSCs compared with BSTs. Consensus clustering highlighted differences between SBOT methylomes and returned subgroups with malignant- or benign-like methylation profiles. Furthermore, a two-loci DNA methylation signature can distinguish between these SBOT subgroups with benign- and malignant-like methylation characteristics. Our findings indicate striking similarities between SBOT and LGSC methylomes, supporting a common origin and the view that LGSC may arise from SBOT. A subgroup of SBOTs can be classified into tumors with a benign- or a malignant-like methylation profile that may help in identifying tumors more likely to progress into LGSCs. (Am J Pathol 2013, 182: 668-677; http://dx.doi.org/10.1016/j.ajpath.2012.11.040)
\end{abstract}

Low-grade serous carcinomas (LGSCs) form a distinct entity of ovarian tumors representing approximately $6 \%$ to $9 \%$ of ovarian carcinomas. ${ }^{1}$ LGSCs are usually confined to the ovary at presentation; their progress is slow and stepwise. ${ }^{2}$ Compared with high-grade serous carcinomas (HGSCs), which account for most ovarian cancers, LGSCs affect women at a younger age and patients have a considerably better prognosis, with a 5-year overall survival rate of approximately $70 \%$ compared with only $40 \%$ for patients with HGSCs. ${ }^{1-3}$ At the molecular level, LGSCs have been shown to harbor mutations in $B R A F$ or $K R A S$, which occur in approximately $50 \%$ of tumors. ${ }^{4}$ However, additional consistent genetic changes have not been identified and, accordingly, the pathogenesis of LGSCs is less well understood. It has been proposed that LGSCs follow a distinct route of molecular pathogenesis, evolving stepwise from benign serous ovarian tumors (BSTs) through serous borderline tumors (SBOTs). ${ }^{5}$ In support of this pathway model are mutations in BRAF and KRAS, occurring in SBOTs at similar frequencies to those observed in LGSCs. ${ }^{6}$ Like LGSCs, SBOTs are a distinct group that accounts for approximately $10 \%$ of ovarian neoplasms. ${ }^{7}$ Six hundred cases occur annually in the United Kingdom, mostly affecting women in the reproductive age group. ${ }^{7}$ Most SBOTs are confined to the ovary at presentation and have an excellent prognosis after surgical removal, with a 5-year overall survival rate for earlystage disease of $98 \%$ and between $86 \%$ and $92 \%$ for more advanced stages of the disease. ${ }^{8}$ Traditionally, SBOTs have been classified as tumors of ovarian surface epithelium origin that arise from BSTs. ${ }^{9,10}$ Recently, it has been proposed that, similar to HGSCs, SBOTs may originate from the epithelium of the fallopian tube, with secondary involvement of the

Supported by Cancer Research UK grant C536/A6689 (C.Z., W.D., and R.B.), Ovarian Cancer Action grant (E.C. and R.B.), Ovacome grant (I.K.-M. and M.E.-B.), the Imperial Experimental Cancer Medicine Centre, and the NIH Research Imperial Biomedical Research Centre. 
ovary. ${ }^{11-13}$ It has been suggested that, because of chronic inflammation, papillary hyperplasia of tubal epithelium may develop, with shedding and implantation of the tubal epithelium on ovarian surfaces, with subsequent development of SBOTs. ${ }^{12}$ In addition to the similar mutation spectrum of SBOTs and LGSCs, recent genome-wide molecular expression profiling studies and DNA methylation profiling analyses also supported a close association between SBOTs and LGSCs. ${ }^{14,15}$ In particular, Shih et $\mathrm{al}^{15}$ characterized the methylation profiles of BSTs, SBOTs, LGSCs, and HGSCs by interrogating $1505 \mathrm{CpG}$ sites using Illumina's GoldenGate arrays (Illumina Inc., San Diego, CA). Their findings demonstrate that HGSCs clearly differ from LGSCs, with LGSCs being more closely related to SBOTs and BSTs. However, the exact relationship between SBOTs and LGSCs is not entirely clear, ${ }^{16}$ and fewer studies have focused on the molecular steps involved in the progression from benign ovarian lesions through SBOTs to LGSCs than on the differences between LGSCs and HGSCs. ${ }^{5}$ Because SBOTs are believed to be the precursors of LGSCs, further characterization of their relationship to invasive carcinoma, and putative benign precursor lesions, may help in elucidating the underlying mechanisms contributing to the genesis of LGSCs.

Aberrant DNA methylation-associated transcriptional silencing is widely observed in cancers. ${ }^{17}$ We have examined genome-wide the DNA methylomes of LGSCs, SBOTs, and BSTs to further characterize the epigenomic relationship between these three subtypes of ovarian tumors. In particular, the current study is aimed at providing further insight into the previously reported epigenomic relationship between SBOTs and LGSCs ${ }^{15}$ and identifying putative methylation markers that might allow distinguishing between subtypes. The study elucidates the differences in DNA methylation of LGSCs, SBOTs, and BSTs and the identification of subgroups of SBOTs that have a benign- or malignant-like methylation profile.

\section{Materials and Methods}

\section{Tumor Samples and Clinicopathological Data}

A total of 16 BSTs, 19 SBOTs, and 12 LGSCs were collected at the Hammersmith Hospital Campus, Imperial College (London, UK). The histopathological diagnosis of all selected cases was reviewed and verified by a specialist gynecological histopathologist (M.E.-B.), according to the established criteria for the diagnosis of the different tumor entities. ${ }^{9}$ In brief, serous tumors showing frank invasion and low-grade nuclear atypia were classified as LGSCs, whereas serous tumors showing borderline changes (eg, complex hierarchical papillary architecture, cellular crowding, and cytological atypia) in $>10 \%$ of the lesion were classified as SBOTs. ${ }^{9}$ Appropriate ethical approval for all samples collected was obtained (Hammersmith and Queen Charlotte's and Chelsea Research Ethics Committee). The age range was 20 to 88 years (median, 68 years) for patients with BSTs, 25 to 82 years (median, 55 years) for patients with SBOTs, and 24 to 61 years (median, 49 years) for patients with LGSCs. All samples had $>70 \%$ epithelial cell content, and no microdissection was undertaken. Of 19 SBOTs, 2 showed a micropapillary pattern, whereas 2 tumors showed microinvasion and 4 tumors were associated with noninvasive implants. Patients with SBOTs have been undergoing follow-up for a median of 5 years (range, 2 to 8 years). None of the patients developed recurrence or disease progression.

\section{DNA Extraction}

DNA was extracted from fresh snap-frozen tissue. Briefly, tissue was homogenized in $180 \mu \mathrm{L}$ of ATL buffer (Qiagen, West Sussex, UK) using a TissueLyser (Tissuelyser I; Retsch, Leeds, UK) at $15 \mathrm{~Hz}$, for 20 seconds. Supernatantcontaining disrupted tissue was transferred to a $1.5-\mathrm{mL}$ tube, $20 \mu \mathrm{L}$ of Proteinase K (Qiagen) was added, and the sample was incubated at $56^{\circ} \mathrm{C}$ overnight. DNA was then extracted using the QIAamp DNA Mini kit (Qiagen), according to the manufacturer's protocol.

\section{DNA Methylation Profiling}

Genome-wide DNA methylation profiling was performed using Infinium HumanMethylation27 BeadChips (Illumina, San Diego, CA), which simultaneously interrogate 27,578 $\mathrm{CpG}$ sites within the promoter regions of $>14,000$ genes $(15,369 \mathrm{CpG}$ sites located within $\mathrm{CpG}$ islands and 12,209 $\mathrm{CpG}$ sites located outside of $\mathrm{CpG}$ islands, as defined by Gardiner-Garden and Frommer $\left.{ }^{18}\right)$. Genomic DNA $(1 \mu \mathrm{g})$ was bisulphite modified using the EZ DNA Methylation Kit (Zymo Research, Irvine, CA). Converted DNA, 200 ng, was further processed to run BeadArrays (Illumina), according to the manufacturer's instructions. Each locus is represented by fluorescent signals from two bead types corresponding to the methylated and unmethylated alleles, respectively. The methylation status $\beta$ ( $\beta$ ) for each individual $\mathrm{CpG}$ locus was calculated as the ratio of fluorescent signals: $\beta=\operatorname{Max}(\mathrm{M}, 0) /$ $[\operatorname{Max}(\mathrm{M}, 0)+\operatorname{Max}(\mathrm{U}, 0)+100]$, using the average probe intensity for the methylated $(\mathrm{M})$ and unmethylated (U) alleles. $\beta$ Ranges from 0 to 1 , with $\beta=1$ indicating complete methylation, whereas $\beta=0$ represents no methylation. The data were assembled using BeadStudio software Methylation Module version 1.0.5 (Illumina), with normalization and background correction per the manufacturer's instructions. Array control probes were used to assess the quality of the samples. Subsequent analysis was performed in $\mathrm{R}$ version 2.10 .1 (http://www.r-project.org).

\section{Unsupervised Clustering}

Unsupervised hierarchical clustering (HCL) was performed using DNA methylation profiles from 16 BSTs, 19 SBOTs, 
and 12 LGSCs using euclidean distance and average linkage in the TIGR MultiExperiment Viewer. ${ }^{19}$

For initial identification of SBOTs having benign- or malignant-like methylation characteristics, further unsupervised HCL of DNA methylation profiles was performed using two different distance metrics and two different clustering algorithms. First, a correlation-based distance metric was calculated for each pair of samples and used as the basis of HCL, defined as 1 - Pearson correlation coefficient between the vectors of methylation values from each sample. Second, HCL was performed using the hclust function provided in $\mathrm{R}$ version 2.10 .1 (http://www.r-project.org) using complete or average linkage. The resulting sample clustering was confirmed by repeating the analyses using a euclidean distance metric, which, in all cases, produced the same structure as the clustering based on the correlation distance metric.

\section{Consensus Clustering}

The designation of SBOTs into groups with benign- or malignant-like methylation profiles was confirmed using consensus clustering. ${ }^{20}$ Consensus clustering was performed with both HCL (using average linkage) and $k$-means, specifying 2 to 10 clusters, for each of a euclidian and 1 Pearson correlation coefficient distance. One hundred clustering runs were performed, each on a different randomly selected subset consisting of $80 \%$ of the samples in the data set. Consensus matrices obtained from clustering runs were imaged as heat maps indicating the proportion of times each pair of samples was found to cluster together, across both HCL and $k$-means clustering. The optimal number of clusters was chosen on the basis of the $\delta-k \operatorname{plot}^{20}$ and the perceived stability of the clustering, as evaluated by inspection of the consensus matrix heat maps. Resamplingbased consensus clustering was performed using the $\mathrm{R}$ package clusterCons, with the hclust and $k$-means clustering functions provided in $\mathrm{R}$.

\section{SBOT Classifier Analysis}

Significantly differentially methylated $\mathrm{CpG}$ sites between consensus subgroups of SBOTs with benign-like $(n=3)$ and malignant-like $(n=6)$ methylation profiles were identified using empirical Bayes moderated $t$-statistics calculated by a linear model for microarray data (LIMMA), ${ }^{21}$ setting a significance threshold at a Benjamini-Hochberg adjusted $P \leq 0.05$. CpG sites with the highest methylation differences between the subgroups of SBOTs having benign- and malignant-like methylation profiles were identified as potential classifiers. These $\mathrm{CpG}$ sites were tested, individually and in combination, to find a minimal subset that could perfectly recapitulate the previously observed clustering of SBOT samples, using complete linkage and euclidean distance after scaling each measurement by the median across the selected samples.

\section{Gene Annotation}

Gene annotation was performed using the March 2006 (National Center for Biotechnology Information/36/hg18) assembly at the University of California, Santa Cruz, database (http://genome.ucsc.edu/cgi-bin/hgGateway?hgsid = 31998234 $3 \&$ clade $=$ mammal \&org $=$ Human $\& d b=h g 18$, last accessed February 1, 2011).

Individual $\mathrm{CpG}$ sites located within a $\mathrm{CpG}$ island, as defined by Gardiner-Garden and Frommer, ${ }^{18}$ were linked to an associated gene if the $\mathrm{CpG}$ island was within a 2-kb distance from the respective transcription start site. ${ }^{22}$ Non-CpG island-related $\mathrm{CpG}$ sites were linked to a gene if they were located within $2 \mathrm{~kb}$ from the transcription start site.

\section{Differential Methylation Analysis}

Differential methylation at $\mathrm{CpG}$ sites was analyzed by a LIMMA comparing between the different subgroups of ovarian tumors (BSTs, SBOTs, and LGSCs). A false-discovery rate $($ FDR $) \leq 0.09$ was used as a cutoff for differential methylation analysis. The difference of six pairs of replicates of the HGSC cell line, PEO1, was calculated as the expected difference (null distribution). The observed difference was calculated from the grouped samples. By using the bootstrap resampling method, we extracted the same number of $\mathrm{CpG}$ sites $(n=27,578)$ from the null distribution 200 times. Each time, an FDR was calculated using the formula given later. The median of FDRs was used as an estimation of the probability that $\mathrm{CpG}$ sites with differential methylation higher than the selected cutoff were identified by chance. This median FDR was set at 0.09 , corresponding to a difference of $\beta|\Delta \beta| \geq 0.25$.

$$
\mathrm{FDR}=\frac{\text { No. of CpG Sites Over Cutoff in Null Data }}{\text { No. of CpG Sites Over Cutoff in Real Data }}
$$

Differentially methylated genes were extracted as showing a significantly increased or decreased $|\Delta \beta| \geq 0.25$ between tumor subgroups analyzed at one or more associated $\mathrm{CpG}$ sites.

\section{Gene Ontology Analysis}

DNA methylation profiles were used for gene ontology analyses using the functional annotation tool in the database, DAVID, as previously described. ${ }^{23}$

\section{Data Deposition}

Methylation profiling data are available in the National Center for Biotechnology Information's Gene Expression Omnibus (http://www.ncbi.nlm.nih.gov/geo; accession number GSE40105).

\section{Bisulphite Modification and Pyrosequencing Analysis}

Genomic DNA $(1 \mu \mathrm{g})$ was bisulphite modified using the EZ DNA Methylation Kit (Zymo Research, Irvine, CA). Pyrosequencing primer sets covering the differentially 


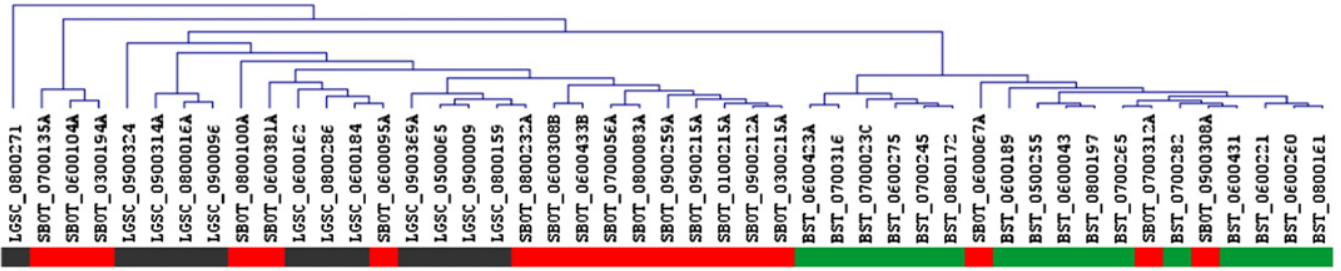

Figure 1 Unsupervised HCL separates between BST and LGSC samples. A cluster dendrogram shows that most SBOTs (red) cluster with LGSCs (gray), with only a small subgroup of three SBOTs being more similar to BSTs (green).

methylated CpG sites in the DLX5 (corresponding to $\operatorname{cg} 01169726$ ) and $C C D C 104$ (corresponding to $\operatorname{cg} 23192899$ ) genes were as follows: DLX5_PYRO_F, 5'-TTGAGGTTTTTGATTGGAATATATTGT-3'; DLX5_PYRO_R, $5^{\prime}$ biotin-ATAACACCCTAACTCCTCCAACTAATTTT- $3^{\prime}$; DLX5_PYRO_S, 5'-TTTGTGTTGTGGTTT-3'; CCDC104_ PYRO_F, 5'-biotin-GGTGTTGGGGATATAATGATGAAT-3'; CCDC104_PYRO_R, 5'-AATTACAATAAACCACTACACTAC-3'; and CCDC104_PYRO_S, 5'-ACCACTACACTACAACCTAAA-3'. Pyrosequencing PCR was performed in duplicate for each sample in a $25-\mu \mathrm{L}$ volume containing $0.2 \mu \mathrm{L}$ of Faststart Taq polymerase (Roche, Welwyn Garden City, UK), $2.5 \mu \mathrm{L}$ of Faststart Buffer (Roche), $0.05 \mathrm{mmol} / \mathrm{L}$ dNTPs (Roche), $0.8 \mu \mathrm{mol} / \mathrm{L}$ primers (each), and $1 \mu \mathrm{L}$ of modified DNA template using the following conditions: $95^{\circ} \mathrm{C}$ for 6 minutes, 35 (CCDC104) or 40 (DLX5) cycles of $95^{\circ} \mathrm{C}$ for 30 seconds, $58^{\circ} \mathrm{C}$ for 30 seconds, and $72^{\circ} \mathrm{C}$ for 30 seconds, followed by $72^{\circ} \mathrm{C}$ for 5 minutes. Pyrosequencing of PCR products was performed using the PyroGold Reagent Kit (Qiagen), according to the manufacturer's instructions. The methylation percentage of $\mathrm{CpG}$ sites for $D L X 5$ and $C C D C 104$ was calculated by using Pyro Q-CpG software version 1.0.9 (Biotage, Uppsala, Sweden) which converts the resulting pyrograms into numerical values for peak heights. The methylation status at each gene in each sample was calculated by averaging values across duplicates.

\section{Results}

DNA Methylation Profiles Can Separate LGSCs from BSTs But Not SBOTs

We analyzed the DNA methylation profiles of $27,578 \mathrm{CpG}$ sites in 16 BSTs, 19 SBOTs, and 12 LGSCs using Infinium HumanMethylation27 BeadChips. Unsupervised HCL of DNA methylation profiles was performed to determine the relationship between these subgroups of tumors. HCL analysis showed distinct separation between BST and LGSC samples that formed separate clusters (Figure 1). Inclusion of SBOTs, however, revealed high similarities between SBOT and LGSC methylomes, with $16(84 \%)$ of 19 SBOTs coclustering with LGSC samples. We also observed 3 (16\%) of 19 SBOTs (0600067A, 0700312A, and 0900308A) clustering together with the subgroup of BSTs, suggesting that there is a subset of SBOTs that shares DNA methylation characteristics with BSTs.

In addition, principal component analysis (PCA) was used to explore the components relevant to the subtypes of ovarian tumors analyzed (Figure 2). By using PC1 and PC2, we observed separate localization of BSTs and LGSCs along PC1, whereas SBOTs ranged from BSTs to LGSCs. PCA, therefore, further supported a clear distinction between LGSCs and BSTs, but not SBOTs. Moreover, it emphasized SBOTs as a diverse group, with individual tumors showing either more malignant- or benign-like disease DNA methylation characteristics.

\section{LGSCs Show Distinctive Hypermethylation and Hypomethylation Patterns Compared With BSTs}

We further used DNA methylation profiles to examine differences between the 12 LGSCs and 16 BSTs, applying LIMMA. ${ }^{21} \mathrm{We}$ analyzed all 27,578 $\mathrm{CpG}$ sites defining differences in DNA methylation as showing a significantly increased or decreased difference of $\beta(|\Delta \beta|)$ corresponding to an FDR of $<0.05$, estimated from biological replicates within the study $(|\Delta \beta| \geq 0.25)$ between the group of LGSCs and the group of BSTs at one or more associated CpG sites (as described in Materials and Methods). By using these criteria, we identified multiple methylation changes with 383 genes (corresponding to $456 \mathrm{CpG}$ sites) (Supplemental Table S1) being hypermethylated and 340 (Materials and

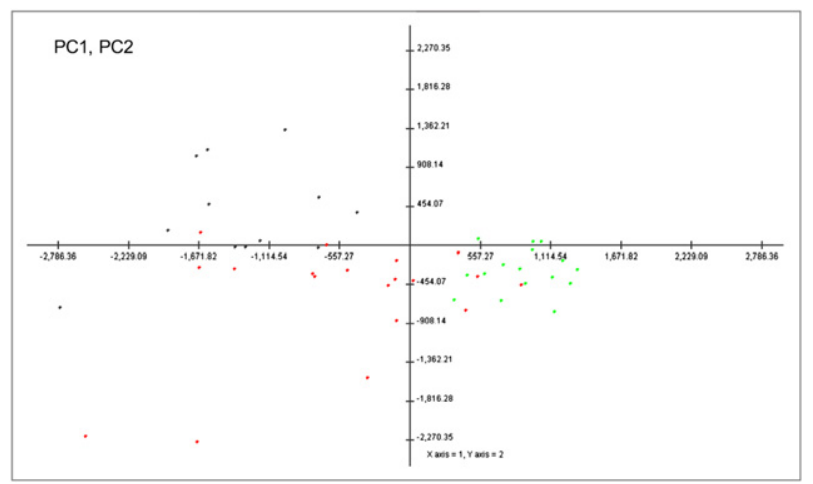

Figure 2 PCA of BSTs, SBOTs, and LGSCs. PC1 and PC2 mainly determine localization of BST, SBOT, and LGSC samples, with only BSTs and LGSCs being clearly separated along PC1. Green, BST samples; red, SBOT samples; gray, LGSC samples. 
Table 1 Significantly Enriched Functional Terms in Genes Associated with Hypermethylated and Hypomethylated CpG Sites in LGSCs vs BSTs at an FDR of $<0.05$

\begin{tabular}{|c|c|c|c|c|c|c|}
\hline Category & Term & No. of genes & $\%$ & Fold enriched & $P$ value* & FDR \\
\hline \multicolumn{7}{|c|}{ Hypermethylated in LGSCs vs BSTs } \\
\hline GOTERM_BP_FAT & G0:0007155 cell adhesion & 42 & 11.1 & 2.6 & 0.00006 & 0.00005 \\
\hline GOTERM_CC_FAT & G0:0005576 extracellular region & 79 & 20.9 & 1.8 & 0.00009 & 0.00037 \\
\hline GOTERM_BP_FAT & G0:0007267 cell-cell signaling & 36 & 9.5 & 2.6 & 0.00084 & 0.00071 \\
\hline GOTERM_CC_FAT & G0:0044421 extracellular region part & 48 & 12.7 & 2.1 & 0.00030 & 0.00133 \\
\hline GOTERM_CC_FAT & G0:0044459 plasma membrane part & 83 & 22.0 & 1.6 & 0.00206 & 0.00904 \\
\hline GOTERM_CC_FAT & G0:0031226 intrinsic to plasma membrane & 54 & 14.3 & 1.9 & 0.00289 & 0.01270 \\
\hline GOTERM_MF_FAT & G0:0032403 protein complex binding & 17 & 4.5 & 3.8 & 0.00603 & 0.01568 \\
\hline \multicolumn{7}{|c|}{ Hypomethylated in LGSCs vs BSTs } \\
\hline GOTERM_CC_FAT & G0:0005576 extracellular region & 72 & 21.5 & 1.9 & 0.00002 & 0.00010 \\
\hline
\end{tabular}

*Bonferroni corrected.

Methods) genes (corresponding to $404 \mathrm{CpG}$ sites) (Supplemental Table S2) being hypomethylated in LGSCs compared with BSTs.

To explore any patterns within the set of genes showing differential methylation at linked $\mathrm{CpG}$ sites in LGSCs versus BSTs, we interrogated gene ontologies ${ }^{24}$ to identify enrichment of gene functions. Table 1 shows highly significant results for genes showing differential methylation at linked CpG sites in LGSCs compared with BSTs, with $P<0.01$ after controlling for an FDR of $<0.05$. Gene ontology analysis revealed significant enrichment of gene groups associated with cell adhesion (FDR $<5 \times 10^{-5}$ ), the extracellular region of a cell (FDR $<3.7 \times 10^{-4}$ ), and cell-cell signaling $\left(\right.$ FDR $\left.<7.1 \times 10^{-4}\right)$ in the set of hypermethylated genes in LGSCs compared with BSTs, which is consistent with a more invasive phenotype of LGSCs (Table 1). In addition, cellular components, such as the plasma membrane (FDR $<2.9 \times$ $10^{-3}$ ) were found among the top 10 of terms. Within the set of hypomethylated genes, we also observed significant overrepresentation of genes linked to the extracellular region of the cell, such as matrix metallopeptidases (FDR $<1 \times 10^{-4}$ ) (Table 1). Notably, none of the hypermethylated genes associated with the extracellular region was contained within the list of hypomethylated genes linked to the extracellular region (Supplemental Tables S3 and S4). We did not observe any further significant enrichment of gene groups among genes associated with hypomethylated $\mathrm{CpG}$ sites.
In contrast to the multiple methylation changes that we observed between LGSCs and BSTs, comparison of LGSC and SBOT samples revealed little significant methylation differences, with only one gene (NTF3, corresponding to one $\mathrm{CpG}$ site) having increased methylation in LGSCs versus SBOTs. This was in agreement with LGSC and SBOT samples showing high similarities, as observed by HCL. We further compared the DNA methylation profiles of SBOTs and BSTs. Differential methylation was observed in SBOTs compared with BSTs, however, to a lesser extent than in LGSCs, with 55 genes (corresponding to $62 \mathrm{CpG}$ sites) (Supplemental Table S5) being hypermethylated and 73 genes (corresponding to $79 \mathrm{CpG}$ sites) (Supplemental Table S6) being hypomethylated. Differences in DNA methylation between SBOTs and BSTs might reflect important steps of progression to LGSCs; however, no enrichment in functional categories was observed at associated genes, as determined by gene ontology analysis.

Because DNA methylation profiles of SBOTs and LGSCs showed high similarity using HCL and LIMMA analysis, we compared the lists of differentially methylated genes in these two subgroups versus BSTs (Figure 3). A high overlap between the set of hypermethylated and hypomethylated genes was found, with 54 of 55 hypermethylated genes in SBOTs being hypermethylated in LGSCs and 72 of 73 hypomethylated genes in SBOTs being contained within the list of hypomethylated genes in LGSCs compared with
LGSC vs BST SBOT vs BST

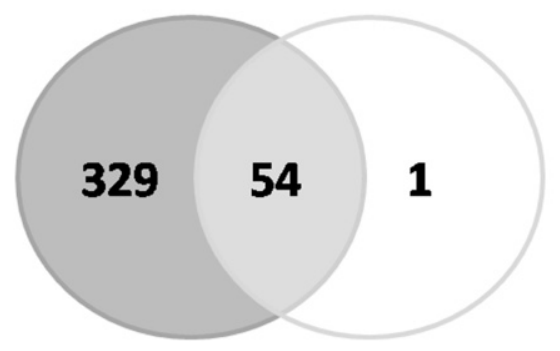

LGSC vs BST SBOT vs BST

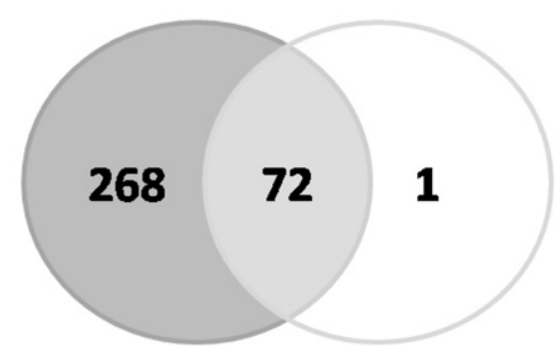

Figure 3 Overlap between hypermethylated and hypomethylated genes in LGSCs versus BSTs and SBOTs versus BSTs shows that DNA methylation changes in SBOT and LGSC tumors are strikingly similar. Left panel: Venn diagram depicts the overlap between hypermethylated genes. Right panel: Venn diagram shows the overlap between hypomethylated genes in LGSCs and SBOTs versus BSTs. 
BSTs (Supplemental Tables S7 and S8). This high degree of overlap further supported the observation that DNA methylation changes in SBOTs and LGSCs are strikingly similar.

\section{A Two-Loci DNA Methylation Signature Can Distinguish between SBOTs With Benign- and Malignant-Like Methylation Characteristics}

LIMMA analysis did not identify significant methylation changes between SBOT and LGSC samples. However, we observed a subgroup of SBOTs coclustering with LGSC samples and a subgroup coclustering with BSTs (Figure 1). To gain more insight into the genes altered by DNA methylation in SBOTs that were more closely related to LGSCs compared with SBOTs with a benign appearance, we defined two groups of SBOTs comprising SBOTs with a benign-like methylation profile and SBOTs with a malignant-like methylation profile. To this end, we first filtered for those SBOT samples that clustered consistently with either LGSCs (SBOTs with a malignant-like methylation profile) or BSTs (SBOTs with a benign-like methylation profile) by HCL using two different distance metrics and two different clustering algorithms (as described in Materials and Methods). This approach returned two subgroups of three SBOTs stably coclustering with BSTs and being defined as showing a benign-like methylation profile (X0600067, X0700312, and X0900308) and six SBOTs that consistently coclustered with LGSC being defined as having a malignant-like methylation profile (X0900215, X0800232, X0600308, X0600433, X0600095, and X0600381) (Supplemental Figure S1).
Furthermore, we confirmed the definition of these two subgroups of SBOTs applying consensus clustering using two different clustering algorithms, HCL and $k$-means clustering (for $k=2$ to $k=10$ groups; as described in Materials and Methods). The resulting consensus matrices supported the partitioning of the data into three main clusters, with BSTs forming a separate cluster away from LGSC samples (Figure 4, A and B, and Supplemental Figure S2). SBOTs cocluster with either of these clusters, substantiating the subgroup of three SBOTs with a benign-like methylation profile consistently segregating together with BST samples, whereas the six SBOTs with malignant-like methylation profiles were stably clustering together with LGSCs (Figure 4A).

A comparison of DNA methylation between these subgroups revealed a small set of two CpG sites, cg01169726 (associated with $D L X 5$ ) and $\operatorname{cg} 23192899$ (associated with CCDC104), that can classify these samples according to their group origin (Figure 5). Low methylation at these two probes was consistently observed in SBOTs with benign-like methylation profiles, whereas at least one of the two probes was found to be methylated in all of the SBOT samples with malignant-like methylation characteristics. We validated these findings using bisulphite pyrosequencing in the samples analyzed (Figure 5 and Supplemental Figure S3). We observed a high correlation between $\beta$ values and methylation percentages, as generated by bisulphite pyrosequencing, with $R^{2}=$ 0.957 indicating that a simple pyrosequencing test would allow to recapitulate differences between SBOTs with different methylation characteristics and to distinguish between these subgroups of SBOTs (Supplemental Figure S4). Although our

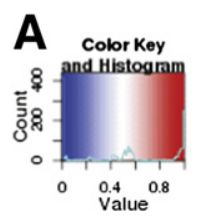

B
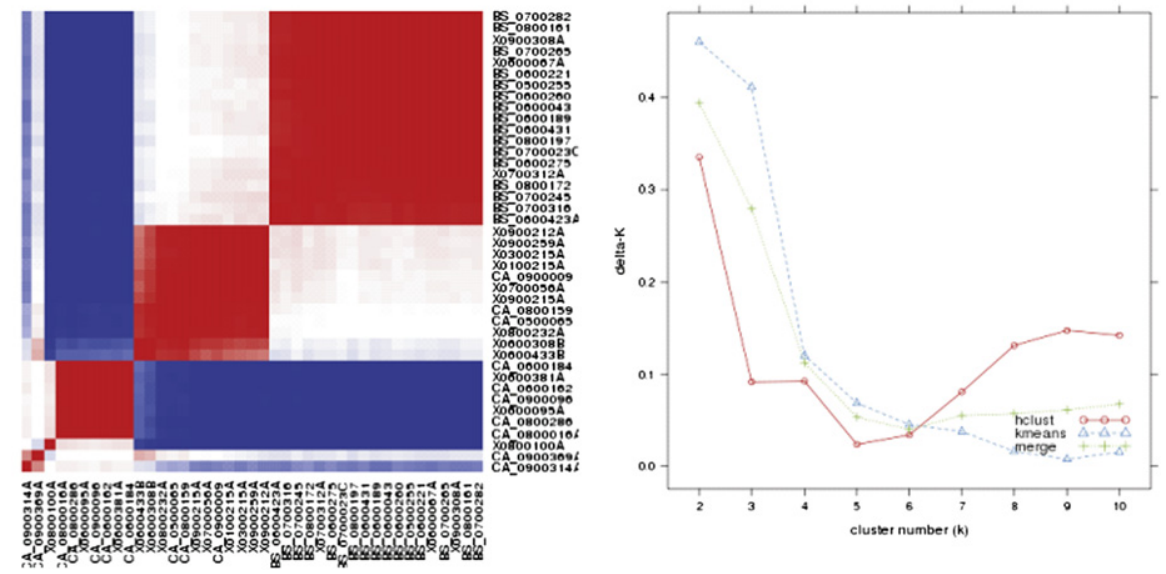

Figure 4 Consensus clustering. A: Merged consensus matrix for $k=3$ and a correlation distance metric. This heat map shows the proportion of 200 resampled clustering runs (each using $80 \%$ of the data set's samples, chosen at random) in which each pair of samples appears in the same cluster; 100 of the clustering runs used $\mathrm{HCL}$, and 100 of the clustering runs used $k$-means clustering. The color bar in the top left of the figure shows the scale, with red indicating that the given pair of samples always appears in the same cluster and blue indicating that the given pair of samples never appears in the same cluster. B: $\delta-k$ Plot indicating an increase in clustering stability with a corresponding increase in the number of clusters, which decreases significantly after $k=3$. 


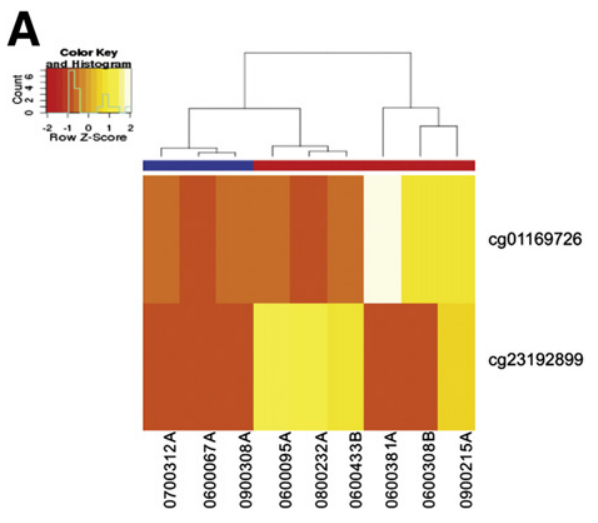

B

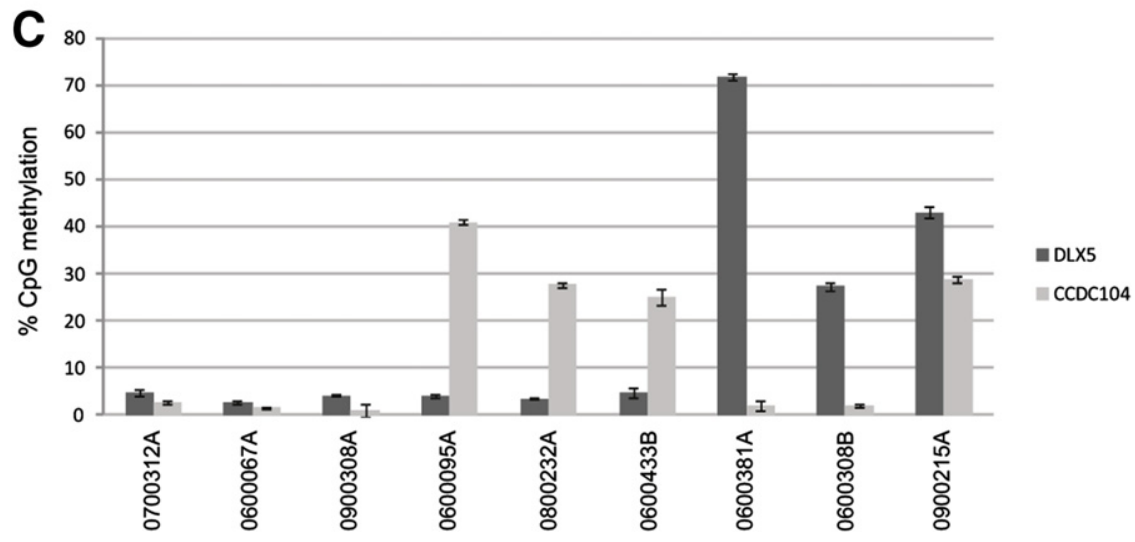

Figure 5 A two-gene signature can separate between SBOTs with benign- and malignant-like methylation profiles. A: Heat map showing methylation levels at the two chosen CpG sites. The $\beta$ values were transformed to standardized $Z$-scores for visualization purposes. At least one of the two CpG sites was methylated in all SBOT samples with malignant-like methylation profiles (red), whereas low methylation was present in all SBOT samples with benign-like methylation profiles (blue) at both CpG sites. B: Cluster dendrogram showing that these two probes can be used to reproduce the classification obtained based on consensus clustering of the genome-wide DNA methylation profiles. C: Validation of the two-gene signature using bisulphite pyrosequencing confirms increased methylation levels in SBOTs with malignant-like methylation profiles in at least one of the two genes (DLX5 or CCDC104). subgroup set of SBOTs was comparatively small, this suggests that it may be possible to predict those SBOTs that are more likely to progress into malignant LGSCs from those with a benign appearance. Prospective validation of this classifier in a bigger group of samples could help in the management of this tumor type, which presents a major challenge.

\section{Discussion}

In this study, we explored the potential progression sequence leading from BSTs via SBOTs to LGSCs and, in particular, epigenetically deregulated genes potentially involved in the mechanisms of transformation from SBOTs to LGSCs. To our knowledge, this is the first study using genome-wide DNA methylation profiling across $>27,000$ $\mathrm{CpG}$ sites to analyze the relationship between these different subtypes of ovarian tumors. Our findings indicate that DNA methylation profiles can distinctly separate between BSTs and LGSCs, whereas they do not distinguish between SBOTs and LGSCs, as demonstrated by unsupervised HCL and PCA. Differences in DNA methylation are more prevalent between LGSCs and BSTs than between SBOTs and BSTs, as the suggested progression sequence between these tumor subtypes, which proceeds from adenoma through SBOT to LGSC, ${ }^{25}$ would predict. Moreover, aberrant DNA methylation of genes with a role in cell adhesion, cell-cell signaling, and the extracellular region was significantly overrepresented in LGSCs compared with BSTs. The identified gene groups contained genes previously reported to be epigenetically deregulated in LGSCs (eg, APC ). ${ }^{26} \mathrm{In}$ addition, our approach identified other genes with a suggested relevance for cancer progression and invasion, such as catenins (CTNNA3), cadherins ( $\mathrm{CDH} 22$ and $\mathrm{CDH} 4)$, protocadherins ( $P C D H 8$ and $P C D H B 15)$, and integrins (ITGB2 and ITGBL1), which have not previously been shown to be hypermethylated in LGSCs. E-cadherin is a prime example of an epithelial tumor suppressing cadherin, inactivation of which has been associated with invasion and metastasis in several tumor types. ${ }^{28}$ It has been suggested that loss of E-cadherin promotes progression by potentiating Wnt signaling, modulating mitogenic signaling events, and influencing cell polarity. ${ }^{27} \mathrm{We}$ also identified two members of the integrin family that have frequently been involved in tumor progression and invasion. For example, loss of integrin $\beta 1$ (ITGB1) was shown to increase the dissemination of tumor cells, thereby increasing malignancy and cell motility, whereas overexpression of this integrin resulted in decreased tumorigenicity of human breast cancer cells. ${ }^{28,29}$ Although much evidence also indicates elevated expression of integrins as a marker of tumor progression, a dual role of integrins as inhibitors and promoters of progression has recently been suggested. ${ }^{30}$ In particular, it will be interesting to further analyze the possible inhibitory role of the integrins identified in this study, which may be altered in expression via aberrant DNA 
methylation events in the initial steps of tumor progression from benign to malignant. The identification of deregulation of these genes might be useful in the search for new drug targets for this type of ovarian cancer. Treatment for LGSCs still presents as difficult because of the generally chemoresistant nature of this tumor type.

In contrast to the clear separation between LGSCs and BSTs, SBOTs were found to represent an intermediate between these two tumor entities of ovarian cancer. Interestingly, our methylation profiling data are in high agreement with previous genome-wide expression profiling analyses, ${ }^{14}$ which highlighted the high degree of similarity between SBOT and LGSC samples. Consistent with the inability of the previous expression profiling to determine a valid classifier distinguishing SBOTs from LGSCs, ${ }^{14}$ we also could not determine a statistically significant classifier between these subgroups of ovarian tumors. Differential methylation comparison of LGSC and SBOT samples revealed little significant differences, with only one gene (NTF3) being hypermethylated in LGSCs versus SBOTs. Neurotrophin 3 is expressed in Sertoli cells and acts as a chemoattractant for cell migration from the mesonephros into the developing testis, a process critical to the early morphological events of testis cord formation. ${ }^{31} \mathrm{Ntf} 3$ also participates in the differentiation and survival of dopaminergic and noradrenergic neurons. ${ }^{32}$ Yet, it is unclear whether this protein fulfils a critical function in ovarian tissues and might be involved in progression from borderline to malignant ovarian tumors or whether this methylation change, although consistent, occurred by chance, either as part of a methylator phenotype or simply as a random silencing event. ${ }^{33}$

The previous studies on SBOTs have focused on DNA methylation status changes of individual candidate genes or groups of genes ${ }^{26,34-36}$; our genome-wide DNA methylation analysis shows that the number of common DNA methylation changes in SBOTs compared with BSTs is moderate, with 55 genes being commonly hypermethylated and 73 genes being commonly hypomethylated. This observation reflects the heterogeneity of SBOTs. The molecular alterations involved in the progression from SBOTs to LGSCs remain unknown. ${ }^{37}$ Although most SBOTs have an excellent overall prognosis, a subset of SBOTs that show aggressive behavior appear to represent a pathological continuum with invasive LGSCs. ${ }^{7}$ Although we observed high similarities between SBOTs and LGSCs using HCL and differential methylation analysis, we also observed a subset of SBOTs being more similar to BSTs. Our results indicate that SBOTs form a distinct entity, with some tumors having a benign- or malignant-like methylation profile independent of most of the histological features. We identified a two-CpG site signature that can distinguish between these subgroups. These two $\mathrm{CpG}$ sites are associated with the DLX5 and CCDC104 genes. DLX5 encodes the distal-less homeobox 5 protein, which belongs to the homeobox family of transcription factors that are primarily expressed during embryonic development. DLX5 plays important roles during bone development and chondrocyte differentiation ${ }^{38}$ and has been shown to act as a positive regulator of MYC, thereby enhancing cell proliferation. $^{39}$ Moreover, hypermethylation of DLX5 occurs with ageing in mesenchymal stromal cells and blood of postmenopausal women. ${ }^{40,41}$ CCDC104 codes for the coiled-coil domain-containing protein 104 with unknown function. CCDC104 is expressed in various human tissues, including the brain, and is localized mainly to the nucleus, but it is also found in the cytoplasm. ${ }^{42}$ Although our subgroup set of SBOTs was comparatively small, this suggests that it may be possible to identify those SBOTs that are more likely to progress into malignant LGSCs. Validation of this two-loci DNA methylation signature in additional samples would be important. The percentage of SBOTs progressing into a more malignant form of the disease is approximately $5 \%$. Therefore, a big data set of $\geq 100$ samples would be required to provide sufficient statistical power to correlate increased methylation at these genes with progression status of patients with SBOTs. Nevertheless, prospective validation of this classifier could provide the basis for a simple assay helping in early identification of SBOTs with higher potential to progress into a malignant form of the disease.

Despite the potential of the identified classifier, we could not classify all of the SBOTs in this study as having either a benign- or a malignant-like methylation profile. We have used a consensus clustering approach to support the classification of SBOTs into having either a malignant- or benignlike methylation pattern based on hierarchical clustering. It is well recognized that hierarchical clustering can lack robustness and stability; therefore, we used a consensus clustering approach to confirm the robustness of our subclassification. We used stringent criteria for confirmation of subgroup membership using information from optimal consensus clustering runs, shown in Supplemental Figure S2, and only subclassified those samples that showed membership in $>80 \%$ of evaluated clustering runs. Based on this approach, the subclassification of only 9 of 19 SBOTs includes those cases that show a stable classification. Because the remaining 10 SBOTs could not be accommodated into either category, it could be argued that there is a subcategory of SBOTs that show more of a mixed methylation pattern between benignand malignant-like.

In conclusion, our results show that the DNA methylomes of LGSCs and SBOTs differ distinctly from BSTs, with high similarities occurring between a subset of SBOTs and LGSCs supporting a common origin of these two subgroups and the view that LGSCs may arise from SBOTs. Significant DNA methylation differences between LGSCs and BSTs were linked to genes potentially associated with a more invasive phenotype. The role of these genes in cell adhesion, cell-cell signaling, and the extracellular region, which are involved in cell-cell recognition, suggests that 
related signaling pathways could be involved in the development and progression of SBOTs. DNA methylation profiles may, therefore, help in elucidating the pathogenetic sequence leading from benign to malignant tumors, potentially leading to the identification of molecular prognostic biomarkers and therapeutic targets for future therapy.

\section{Supplemental Data}

Supplemental material for this article can be found at http://dx.doi.org/10.1016/j.ajpath.2012.11.040.

\section{References}

1. Seidman JD, Yemelyanova A, Cosin JA, Smith A, Kurman RJ: Survival rates for International Federation of Gynecology and Obstetrics Stage III Ovarian Carcinoma by cell type: a study of 262 unselected patients with uniform pathologic review. Int $\mathrm{J}$ Gynecol Cancer 2012, 22:367-371

2. Vang R, Shih IeM, Kurman RJ: Ovarian low-grade and high-grade serous carcinoma: pathogenesis, clinicopathologic and molecular biologic features, and diagnostic problems. Adv Anat Pathol 2009, 16: 267-282

3. Seidman JD, Horkayne-Szakaly I, Cosin JA, Ryu HS, Haiba M, Boice CR, Yemelyanova AV: Testing of two binary grading systems for FIGO stage III serous carcinoma of the ovary and peritoneum. Gynecol Oncol 2006, 103:703-708

4. Jones S, Wang TL, Kurman RJ, Nakayama K, Velculescu VE, Vogelstein B, Kinzler KW, Papadopoulos N, Shih IeM: Low-grade serous carcinomas of the ovary contain very few point mutations. J Pathol 2012, 226:413-420

5. Shih IeM, Kurman RJ: Ovarian tumorigenesis: a proposed model based on morphological and molecular genetic analysis. Am J Pathol 2004, 164:1511-1518

6. Shih IeM, Kurman RJ: Molecular pathogenesis of ovarian borderline tumors: new insights and old challenges. Clin Cancer Res 2005, 11: 7273-7279

7. Jones MB: Borderline ovarian tumors: current concepts for prognostic factors and clinical management. Clin Obstet Gynecol 2006, 49: $517-525$

8. Cadron I, Leunen K, Van Gorp T, Amant F, Neven P, Vergote I: Management of borderline ovarian neoplasms. J Clin Oncol 2007, 25 : 2928-2937

9. Seidman JD, Cho KR, Ronnett BM, Kurman RJ: Surface epithelium tumors of the ovary. Blaustein's Pathology of the Female Genital Tract, ed 6. Edited by Kurman RJ, Ellenson LH, Ronnett BM. New York, Springer-Verlag, 2011, pp 679-784

10. Seidman JD, Kurman RJ: Subclassification of serous borderline tumors of the ovary into benign and malignant types: a clinicopathologic study of 65 advanced stage cases. Am J Surg Pathol 1996, 20: 1331-1345

11. Kindelberger DW, Lee Y, Miron A, Hirsch MS, Feltmate C, Medeiros F, Callahan MJ, Garner EO, Gordon RW, Birch C, Berkowitz RS, Muto MG, Crum CP: Intraepithelial carcinoma of the fimbria and pelvic serous carcinoma: evidence for a causal relationship. Am J Surg Pathol 2007, 31:161-169

12. Kurman RJ, Vang R, Junge J, Hannibal CG, Kjaer SK, Shih IeM: Papillary tubal hyperplasia: the putative precursor of ovarian atypical proliferative (borderline) serous tumors, noninvasive implants, and endosalpingiosis. Am J Surg Pathol 2011, 35:1605-1614

13. Laury AR, Ning G, Quick CM, Bijron J, Parast MM, Betensky RA, Vargas SO, McKeon FD, Xian W, Nucci MR, Crum CP: Fallopian tube correlates of ovarian serous borderline tumors. Am J Surg Pathol 2011, 35:1759-1765
14. Bonome T, Lee JY, Park DC, Radonovich M, Pise-Masison C, Brady J, Gardner GJ, Hao K, Wong WH, Barrett JC, Lu KH, Sood AK, Gershenson DM, Mok SC, Birrer MJ: Expression profiling of serous low malignant potential, low-grade, and high-grade tumors of the ovary. Cancer Res 2005, 65:10602-10612

15. Shih IeM, Chen L, Wang CC, Gu J, Davidson B, Cope L, Kurman RJ, Xuan J, Wang TL: Distinct DNA methylation profiles in ovarian serous neoplasms and their implications in ovarian carcinogenesis. Am J Obstet Gynecol 2010, 203:584.e1-584.e22

16. Ortiz BH, Ailawadi M, Colitti C, Muto MG, Deavers M, Silva EG, Berkowitz RS, Mok SC, Gershenson DM: Second primary or recurrence? comparative patterns of p53 and K-ras mutations suggest that serous borderline ovarian tumors and subsequent serous carcinomas are unrelated tumors. Cancer Res 2001, 61:7264-7267

17. Esteller M: Epigenetics in cancer. N Engl J Med 2008, 358: $1148-1159$

18. Gardiner-Garden M, Frommer M: CpG islands in vertebrate genomes. J Mol Biol 1987, 196:261-282

19. Saeed AI, Sharov V, White J, Li J, Liang W, Bhagabati N, Braisted J, Klapa M, Currier T, Thiagarajan M, Sturn A, Snuffin M, Rezantsev A, Popov D, Ryltsov A, Kostukovich E, Borisovsky I, Liu Z, Vinsavich A, Trush V, Quackenbush J: TM4: a free, open-source system for microarray data management and analysis. Biotechniques 2003, 34:374-378

20. Simpson TI, Armstrong JD, Jarman AP: Merged consensus clustering to assess and improve class discovery with microarray data. BMC Bioinformatics 2010, 11:590

21. Smyth GK: Linear models and empirical bayes methods for assessing differential expression in microarray experiments. Stat Appl Genet Mol Biol 2004, 3. Article3

22. Saxonov S, Berg P, Brutlag DL: A genome-wide analysis of $\mathrm{CpG}$ dinucleotides in the human genome distinguishes two distinct classes of promoters. Proc Natl Acad Sci U S A 2006, 103:1412-1417

23. Huang da W, Sherman BT, Lempicki RA: Systematic and integrative analysis of large gene lists using DAVID bioinformatics resources. Nat Protoc 2009, 4:44-57

24. Ashburner M, Ball CA, Blake JA, Botstein D, Butler H, Cherry JM, Davis AP, Dolinski K, Dwight SS, Eppig JT, Harris MA, Hill DP, IsselTarver L, Kasarskis A, Lewis S, Matese JC, Richardson JE, Ringwald M, Rubin GM, Sherlock G, Gene Ontology Consortium: Gene ontology: tool for the unification of biology. Nat Genet 2000, 25:25-29

25. Wong KK, Tsang YT, Deavers MT, Mok SC, Zu Z, Sun C, Malpica A, Wolf JK, Lu KH, Gershenson DM: BRAF mutation is rare in advanced-stage low-grade ovarian serous carcinomas. Am J Pathol 2010, 177:1611-1617

26. Tam KF, Liu VW, Liu SS, Tsang PC, Cheung AN, Yip AM, Ngan HY: Methylation profile in benign, borderline and malignan ovarian tumors. J Cancer Res Clin Oncol 2007, 133:331-341

27. Jeanes A, Gottardi CJ, Yap AS: Cadherins and cancer: how does cadherin dysfunction promote tumor progression? Oncogene 2008, 27 : 6920-6929

28. Kren A, Baeriswyl V, Lehembre F, Wunderlin C, Strittmatter K, Antoniadis H, Fassler R, Cavallaro U, Christofori G: Increased tumor cell dissemination and cellular senescence in the absence of beta1integrin function. EMBO J 2007, 26:2832-2842

29. Zutter MM, Santoro SA, Staatz WD, Tsung YL: Re-expression of the alpha 2 beta 1 integrin abrogates the malignant phenotype of breast carcinoma cells. Proc Natl Acad Sci U S A 1995, 92:7411-7415

30. Desgrosellier JS, Cheresh DA: Integrins in cancer: biological implications and therapeutic opportunities. Nat Rev Cancer 2010, 10 : $9-22$

31. Clement TM, Bhandari RK, Sadler-Riggleman I, Skinner MK: SRY directly regulates the neurotrophin 3 promoter during male sex determination and testis development in rats. Biol Reprod 2011, 85: 277-284

32. Cho SC, Kim HW, Kim BN, Kim JW, Shin MS, Cho DY, Chung S, Jung SW, Yoo HJ, Chung IW, Chung US, Son JW: Neurotrophin-3 gene, 
intelligence, and selective attention deficit in a Korean sample with attention-deficit/hyperactivity disorder. Prog Neuropsychopharmacol Biol Psychiatry 2010, 34:1065-1069

33. Issa JP: $\mathrm{CpG}$ island methylator phenotype in cancer. Nat Rev Cancer 2004, 4:988-993

34. Choi YL, Kang SY, Shin YK, Choi JS, Kim SH, Lee SJ, Bae DS, Ahn G: Aberrant hypermethylation of RASSF1A promoter in ovarian borderline tumors and carcinomas. Virchows Arch 2006, 448:331-336

35. Wiley A, Katsaros D, Chen H, Rigault de la Longrais IA, Beeghly A, Puopolo M, Singal R, Zhang Y, Amoako A, Zelterman D, Yu H: Aberrant promoter methylation of multiple genes in malignant ovarian tumors and in ovarian tumors with low malignant potential. Cancer 2006, 107:299-308

36. Kang S, Dong SM, Park NH: Frequent promoter hypermethylation of TGFBI in epithelial ovarian cancer. Gynecol Oncol 2010, 118:58-63

37. Kuo KT, Guan B, Feng Y, Mao TL, Chen X, Jinawath N, Wang Y, Kurman RJ, Shih IeM, Wang TL: Analysis of DNA copy number alterations in ovarian serous tumors identifies new molecular genetic changes in low-grade and high-grade carcinomas. Cancer Res 2009, 69:4036-4042
38. Bendall AJ, Hu G, Levi G, Abate-Shen C: Dlx5 regulates chondrocyte differentiation at multiple stages. Int J Dev Biol 2003, 47: 335-344

39. Xu J, Testa JR: DLX5 (distal-less homeobox 5) promotes tumor cell proliferation by transcriptionally regulating MYC. J Biol Chem 2009, 284:20593-20601

40. Bork S, Pfister S, Witt H, Horn P, Korn B, Ho AD, Wagner W: DNA methylation pattern changes upon long-term culture and aging of human mesenchymal stromal cells. Aging Cell 2010, 9:54-63

41. Teschendorff AE, Menon U, Gentry-Maharaj A, Ramus SJ, Weisenberger DJ, Shen H, Campan M, Noushmehr H, Bell CG, Maxwell AP, Savage DA, Mueller-Holzner E, Marth C, Kocjan G, Gayther SA, Jones A, Beck S, Wagner W, Laird PW, Jacobs IJ, Widschwendter M: Age-dependent DNA methylation of genes that are suppressed in stem cells is a hallmark of cancer. Genome Res 2010, 20: 440-446

42. Totland C, Bredholt G, Haugen M, Haukanes BI, Vedeler CA: Antibody to CCDC104 is associated with a paraneoplastic antibody to CDR2 (anti-Yo). Cancer Immunol Immunother 2010, 59: 231-237 